\title{
Cloned Microglias with Novel Delivery System in Multiple Sclerosis
}

\section{Abdul Mannan Baig*}

Department of Biological and Biomedical Sciences, Aga Khan University, Karachi, Pakistan

\begin{abstract}
Multiple Sclerosis (MS) is a chronic inflammatory neurological disease of the Central Nervous System (CNS), characterized by demyelination and activation of microglia. Mitochondrial mutations and dysfunctions in microglial cells are thought to contribute to the detrimental effects of neuroinflammation seen in MS. The Somatic Nuclear Transfer (SCNT) technology offers a more practical mode of therapy in MS, this method would attempt to dilute and/or progressively replace the mutated and activated microglia with cloned Olfactory Ensheathing Cells (OEC) with remyelinating and scavenging properties which would attempt to limit the progression of MS. Applying SCNTderived Embryonic Stem (ES) cells based therapy by cloning Olfactory Ensheathing Cells (OEC), engineered with an autologous nuclear component of the recipient OEC with a healthy donor oocyte. The inner cell mass of the subsequently developed blastocyst would be the source to generate the radial microglia to be used for cell therapy in MS. The novel proposed transcribrial route device offers a painless mode of cell transplantation to the brain. This mode of generating cloned glia and its transplantation to the brain is expected to replace the mutated and activated microglia of the patients with MS and use the regenerative and remyelinating and scavenging properties of the OEC's, as has been seen in clinical trials in patients with spinal cord injuries. The use of SCNT to develop isogenic ES cellbased therapies for the prevention and treatment of MS associated with mtDNA mutations may open a new avenue of designer's targeted cell therapy unique for the patients with MS. The proposed "transcribrial device" to access the brain can be an advantageous route of delivery of cloned cells to the brain.
\end{abstract}

Keywords: Multiple Sclerosis; OEC's; Neurodegenerative diseases; Stem cell therapy; Regenerative medicine; SCNT

\section{Introduction}

Multiple sclerosis (MS) is a chronic inflammatory neurological disease of the central nervous system; characterized by demyelination, neurodegeneration and astroglial proliferation [1], with complex genetic factors exerting a profound influence [2]. Despite major advances in the current understanding of the pathogenesis of MS, the inflammatory cascade involved in MS remains unknown; although there is considerable evidence implicating the involvement of mitochondria DNA mutations involved in axonal and glial injury mechanisms [35]. Mitochondrial dysfunction in microglial cells has been observed in several animal models of neurodegeneration and aging, in this regard the mitochondrial DNA damage in particular is most pronounced within microglial cells, especially compared to neurons [6]. There is currently no treatment for the loss of neuronal function after damage to the nervous system, multiple attempts at nerve re-growth across the peripheral nervous system (PNS) and (CNS) transition have not been successful [7]. Injuries and neurodegenerative (ND) diseases of the central nervous system (CNS), like MS are unique in that they almost always get repaired by gliosis leading to loss of neurological function and resultant neurological deficits in the affected individual. Widespread microglial activation is a histopathological hallmark of progressive MS and inhibition of this aspect of innate immunity could potentially have beneficial effects on both progressive and relapsing forms of MS [8]. Majority of ND, including (MS) is characterized by activation of microglia and mitochondrial dysfunction in microglial cells are thought to contribute to the harmful effects neuroinflammation seen in these diseases [6]. One of the most distressing aspects of MS treatment is the failure of development of disease-modifying therapies that slow disability in progressive forms of MS. At present, none of the drugs do attempt to reduce the inflammatory process and target either the primary progressive or secondary progressive MS [8]. Very trivial studies in animal models have come up in the field of cell therapy as a treatment modality for this demyelinating disease.

\section{Current Scenarios in the Drug Management of Multiple Sclerosis}

As a result of the past 20 years of research done for treatment of MS, various forms of auto injectable interferon beta (IFN b) and Glatiramer acetate (GA) got into the market with GA $40 \mathrm{mg}$ given subcutaneously reduced the relapse rate at 1 year [9] and became widely used as first line disease treatments. Natalizumab, and to a much lesser extent Mitoxantrone, are second-line intravenously infused treatments. With teriflunomide in patients with relapsing MS study showed teriflunomide's clinical efficacy in reducing the relapse rate and neurological deficits in MS over a period of 2 years [10]. Several phase III studies in relapsing MS are ongoing for second-generation sphingosine-1-phosphate receptor 1 immunomodulators which show promise in reducing some of the side effects associated with such an agent like bradycardia, abnormal hepatic enzyme elevations, and lung fibrosis [8]. Vitamin D deficiency has been recently identified as a risk factor and its potential in the possible disease modifying role and awaits clinical trials in the relapsing MS [11].

\section{Current Status of Stem Cell Therapy in Multiple Sclerosis}

In humans, neurogenesis largely ceases during adulthood, but in two areas of the brain, the hippocampus and olfactory bulb, there is strong evidence of regeneration of substantial numbers of new neurons

*Corresponding author: Abdul Mannan Baig, Department of Biological and Biomedical Sciences, Aga Khan University, Stadium Road, Karachi, Pakistan, Tel: 92-(0)21-3486-4435; Fax: 92-(0)21-3493-4294; E-mail: abdul.mannan@aku.edu

Received July 10, 2014; Accepted December 06, 2014; Published December 08, 2014

Citation: Baig AM (2014) Cloned Microglias with Novel Delivery System in Multiple Sclerosis. J Stem Cell Res Ther 4: 252. doi:10.4172/2157-7633.1000252

Copyright: (c) 2014 Baig AM. This is an open-access article distributed under the terms of the Creative Commons Attribution License, which permits unrestricted use, distribution, and reproduction in any medium, provided the original author and source are credited. 
[12]. Studies done in detail on the use of (ESCs) in MS have revealed that the actual limitation is the preparation of sufficient human oligodendrocytes precursor cells in getting tissue-specific progenitor cells from human embryonic stem cells (ESCs) and the dangers that there are always risk of tumorogenesis in neural cells derived from ESCs, limiting the potentialities of science and therapy in such studies [13]. Another shortcoming of the autologous MSC and induced pluripotent stem cells (iPS), transplantation in the treatment of MS is that of carrying the inherited (mtDNA) mutation to the CNS. For MSC there is lingering doubt whether the MSC derived neurons are functional [14], and as similar fate could be expected if it ends up differentiating into a glia.

\section{Recommendation for a Novel Cell Therapy in Multiple Sclerosis}

From extensive reviews on the cell therapy methodologies it can be computed that partial successes or disappointments encountered in treating MS with autologous embryonic stem cell transplants have occurred due to immune rejection and/or persistence of inherited or acquired mutated mitochondrial DNA in the glial cells in the case of autologous iPS transplants. Although transplantation of Mesenchymal Stem Cell (MSC), or induced pluripotent stem (iPS) cells generated from autologous somatic OECs and reprogrammed into ES-like cells may provide an additional source of autologous stem cells for treating MS, both would be of no potential benefit as would continue to harbour the same mitochondrial mutations (mtDNA) as possessed by glia in CNS [15]. The field of mitochondrial-targeted therapeutics is one that is growing fast and is of great importance and an increasing evidence points towards mutation leading to altered mitochondrial trafficking and fusion-fission dynamics in several ND diseases including MS [16]. MS can be targeted for slowing down the progression, prevention of onset and possible treatment by perhaps by the proposed genetically modified glial cells cloned by somatic Nucleus Transfer (NT) of Olfactory ensheathing cells to a healthy donor oocyte devoid of mtDNA mutations. OECs normally perform regenerative, scavenging and innate immune functions in the olfactory region and have been investigated thoroughly in related to spinal cord injuries, amyotrophic lateral sclerosis and other ND diseases where research suggests that these cells possess a unique ability to remyelinate injured neurons [17]. A new strategy for isolating oligodendrocyte progenitor cells of the human brain may advance the goal of therapeutic remyelination [18].

Here we propose glia cloned with an autologous nuclear component of the recipient's OEC's, union by combination of whole somatic OECs with a healthy oocyte cytosol [15] (Figure 1), offers an alternate speculative likelihood of producing NT-derived ES cells in which mutated mtDNA is substantially diluted/destroyed by the mtDNA of oocyte as it does to the mitochondrial DNA of the spermatozoa, shortly after fertilization. The resulting inner cell mass from the blastocyst stage of development would become a source of induced pluripotent stem cells that would differentiate into a radial glia like OEC's with the inherent properties of axonal and myelin generation and scavenging functions. Xenotranplantation of myelin-forming OEC's from pigs genetically altered to reduce the hyperacute response in humans are able to induce elongative axonal regeneration and remyelination and restore impulse conduction across the transected spinal cord [19]. The transplantation of the proposed cells followed by the self-renewal of this ESC at sites of demyelination would attempt to repair the gliosis and dilute the activated microglia population that is the leading cause of defects seen in MS.

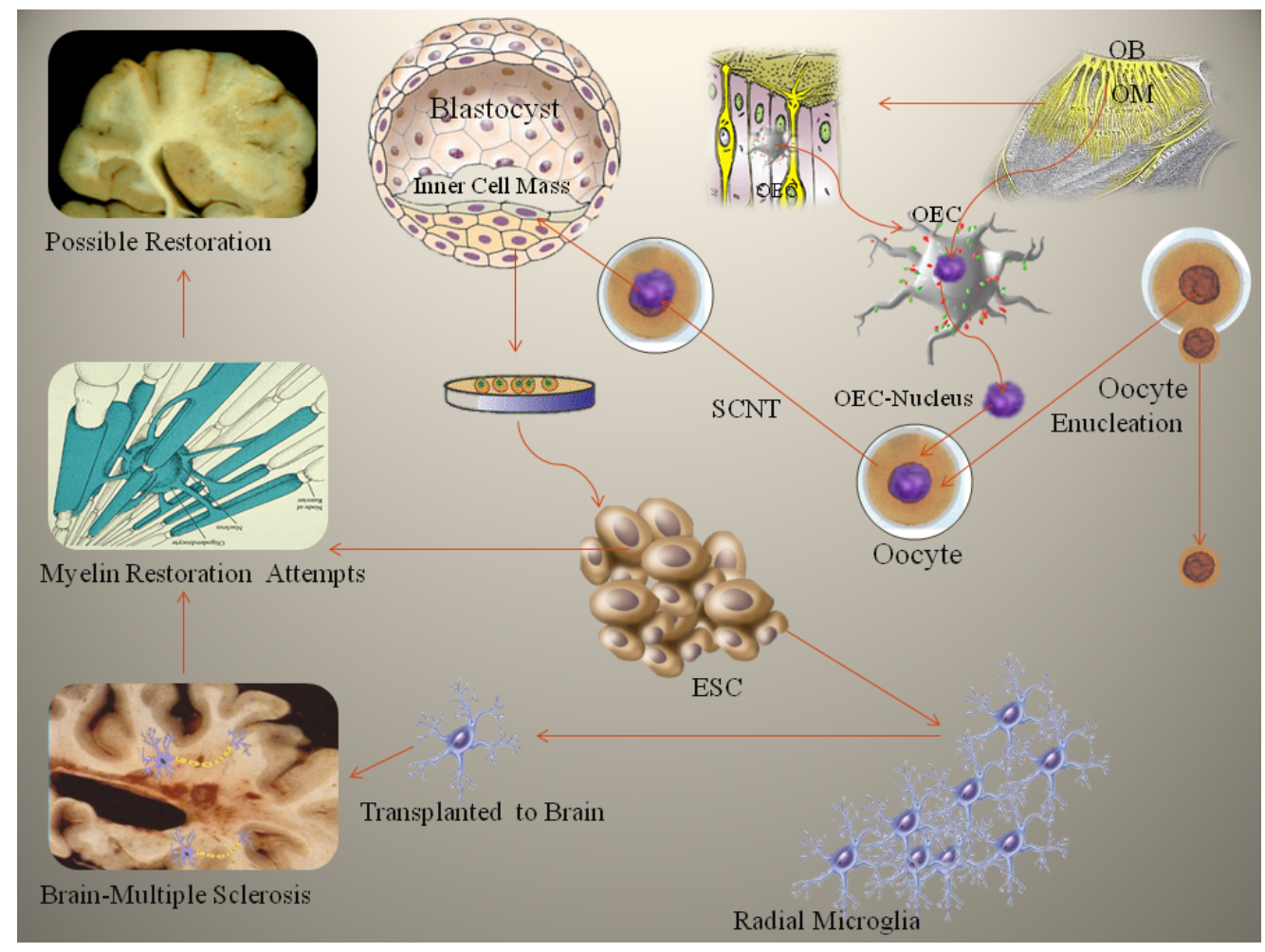

Figure 1: Olfactory Mucosa (OM) and Olfactory Bulb (OB) contain Olfactory Ensheathing Cells (OECs). The single Somatic Nuclear Transfer (SCNT) to an enucleated Oocyte would develop into a blastocyst. The inner cell mass differentiating into radial microglia could be used for transplantation into the central nervous system in Multiple Sclerosis. 
After being reported that transplantation of heterologous fetal OECs into the frontal lobes it has been seen to slow down the rate of clinical progression in ALS patients, the subsequent neuropathologic analysis did not support a therapeutic efficacy for ALS patients even after OEC transplantation via the ventricles, although transplantation of OECs engineered to produce neurotrophins might be helpful to improve the condition in ALS [17]. Culturing and transplanting this cloned glia that expresses identical HLA antigens would not evoke an autoimmune rejection while the mitochondria's of oocyte of a healthy donor would possibly dilute the activated microglia slow down the ongoing neuronal degeneration $[6,15,16]$. The additional rewarding benefit of such transplantation would be that the regenerative and scavenging potentials of OECs in contrast to the over reactive microglia present in the CNS of the MS patients, would help recover the damage already caused by the degenerative process, a feature missing in mesenchymal stem cells (MSC) and iPS. The use of the NT to develop isogenic ES cell-based therapies for the prevention and treatment of MS associated with mtDNA mutations may open a new avenue of designer's targeted cell therapy unique for the patients with MS.

\section{Weighing the Benefits Ahead of Transplantations}

The nuclear profiling of the cloned glia by microarray for differential expression of mtDNA and nuclear DNA of these engineered cells prior to transplant would clearly highlight the benefits of the planned transplantation. A similar gene profiling of the patient's glial cells would help understand the differences in their epigenetic expression and the gains of the procedure in the ongoing MS in the patients. Once evaluated and approved for transplant, these cloned cells like their parent OEC's can be labelled and tracked by a magnetic resonance imaging (MRI) device when being dispersed in the CNS. A recent study has made use of a novel type of Micron-sized Particles of Iron Oxide (MPIO) to label and track these transport-mediated cells via MRI [20].

\section{Novel Delivery via transcribrial route device}

The advantage of transplantation techniques done in past via intrathecal, intraventricular, intra-parenchyma and several other routes can be considered for the aforementioned cloned glial cells. Methods of cells being delivered to CNS through an intranasal route has been reported in the past. The nasal route has therapeutic effects that appear to be equivalent to those induced by intravenous route adult neural stem cells (NSCs) and lead to protection from CNS inflammation and the promotion of remyelination and neural repair. Such an effective alternative route of administration could significantly improve the efficacy of NSC-based therapy in MS [21]. There is potential of this intranasal route principally for the therapy of neurodegenerative disorders, this delivery method provides the option of chronic treatment which may enhance the number of delivered cells in order to achieve therapeutic benefit [22].

We have recently proposed a "transcribrial route device" that is composed of three connected components [23] to access the brain across the criribriform plate of the ethimoid bone. This can be of advantage because of being painless and the trimmed ball-valve terminal could prevent any regurgitation back into the delivery system. A local anaesthetic application prior to the cell therapy could minimize the reflex sneezing that is expected on insertion of the nasal components. Unlike other routes this route would clearly avoid the risks of escape of the cells into the lungs and various neurological complications of other cell therapy delivery systems.

\section{Conflict of Interest}

The author declares that he has no conflict of interest with any institution and/or financial organization regarding the material discussed in the manuscript.

\section{Acknowledgments}

I am indebted to the enormous inspiration I have received from Prof. Naveed Ahmed Khan, who encouraged me to write this manuscript and Dr.HR Ahmad for participation in weekly scholarly meetings at AKU who gave his feedback and support on this proposal. I am thankful to Myra Mannan and Misbah Mannan for their helpful discussions that helped in shaping this manuscript.

\section{References}

1. Martin R, McFarland HF, McFarlin DE (1992) Immunological aspects of demyelinating diseases. Annu Rev Immunol 10: 153-187. [PubMed]

2. Ban M, Elson J, Walton A, Turnbull D, Compston A, et al. (2008) Investigation of the role of mitochondrial DNA in multiple sclerosis susceptibility. PLoS One 3: e2891. [PubMed]

3. Friese MA, Schattling B, Fugger $L$ (2014) Mechanisms of neurodegeneration and axonal dysfunction in multiple sclerosis. Nat Rev Neurol 10: 225-238. [PubMed]

4. Carvalho KS (2013) Mitochondrial Dysfunction in Demyelinating Diseases Original Research Article. Seminars in Pediatric Neurology 20: 194-201. [PubMed]

5. Dutta R, McDonough J, Yin X, Peterson J, Chang A et al. (2006) Mitochondria dysfunction as a cause of axonal degeneration in multiple sclerosis patients. Ann Neurol 59: 478-489. [PubMed]

6. Ferger Al, Campanelli L, Reimer V, Muth KN, Merdian I, et al. (2010) Effects of mitochondrial dysfunction on the immunological properties of microglia. Journal of Neuroinflammation 7: 45. [PubMed]

7. Recknor JB, Mallapragada SK (2006) Nerve Regeneration: Tissue Engineering Strategies, in The Biomedical Engineering Handbook: Tissue Engineering and Artificial Organs. Taylor \& Francis, New York.

8. Cree BA (2014) Multiple Sclerosis Therapeutic Update 2013 in Retrospect A Review of Important Clinical Research in Inpatient Neurology. The Neurohospitalist 4: 63-65.

9. Khan O, Rieckmann P, Boyko A, Selmaj K, Zivadinov R, et al. (2013) Three times weekly Glatiramer acetate in relapsing and remitting multiple sclerosis. Ann Neurol 73: 705-713. [PubMed]

10. Miller A, Kappos L, Comi G (2013) Teriflunomide Efficacy and Safety in Patients with Relapsing Multiple Sclerosis: Results from TOWER, a Second, Pivotal, Phase 3 Placebo-Controlled Study.

11. Ascherio A, Munger KL, White R, Köchert K, Simon KC, et al. (2014) Vitamin $\mathrm{d}$ as an early predictor of multiple sclerosis activity and progression. JAMA Neurol 71: 306-314. [PubMed]

12. Nowakowski RS (2006) Stable neuron numbers from cradle to grave. Proc Nat Acad Sci USA 103: 12219. [PubMed]

13. Ardeshiry Lajimi A, Hagh MF, Saki N, Mortaz E, Soleimani M, et al. (2013) Feasibility of Cell Therapy in Multiple Sclerosis: A Systematic Review of 83 Studies. Int J Hematol Oncol Stem Cell Res 7: 15-33. [PubMed]

14. Franco Lambert AP, Fraga Zandonai A, Bonatto D, Cantarelli Machado D Pêgas Henriques JA (2009) Differentiation of human adipose-derived adult stem cells into neuronal tissue: Does it work? Differentiation 77: 221-228. [PubMed]

15. Baig AM (2014) Designer's microglia with novel delivery system in neurodegenerative diseases. Med Hypotheses 83: 510-512. [PubMed]

16. Johri A, Beal MF (2012) Mitochondrial Dysfunction in Neurodegenerative Diseases. Pharmacol Exp Ther 342: 619-630. [PubMed]

17. Su Z, He C (2010) Olfactory ensheathing cells: Biology in neural development and regeneration. Prog Neurobiol 92: 517-532. [PubMed]

18. Miller RH, Tesar PJ (2011) Tracking down the human myelinating cell. Nat Biotechnol 29: 881-883. [PubMed]

19. Imaizumi T, Lankford KL, Burton WV, Fodor WL, Kocsis JD (2000) 
Citation: Baig AM (2014) Cloned Microglias with Novel Delivery System in Multiple Sclerosis. J Stem Cell Res Ther 4: 252. doi:10.4172/21577633.1000252

Xenotransplantation of transgenic pig olfactory ensheathing cells promotes axonal regeneration in rat spinal cord. Nat Biotechnol 18: 949-953. [PubMed]

20. Sandvig I, Hoang L, Sardella TCP, Barnett SC, Brekken C, et al. (2012) Labeling of olfactory ensheathing cells with micron-sized particles of iron oxide and detection by MRI. Contrast Media Mol Imaging 7: 403-410. [PubMed]

21. Danielyan L, Schafer R, von Ameln-Mayerhofer A, Buadze M, Geisler J, et al. (2009) Intranasal delivery of cells to the brain. Eur J Cell Biol 88: 315-324. [PubMed]
22. Wu S, Li K, Yan Y, Gran B, Han Y, et al. (2013) Intranasal Delivery of Neural Stem Cells: A CNS-specific, Non-invasive Cell-based Therapy for Experimental Autoimmune Encephalomyelitis. J Clin Cell Immunol 4: 142. [PubMed]

23. Baig AM, Khan NA (2014) Novel Chemotherapeutic Strategies in the Management of Primary Amoebic Meningoencephalitis Due to Naegleria fowleri. CNS Neurosci Ther 20: 289-290. [PubMed] 